Journal of Computer Science 4 (4): 327-332, 2008

ISSN 1549-3636

(C) 2008 Science Publications

\title{
Magnetic and Thermal Analysis of Current Transformer in Normal and Abnormal Conditions
}

\author{
${ }^{1}$ M.B.B. Sharifian, ${ }^{1}$ M. Farrokhifar, ${ }^{3}$ R. Esmaeilzadeh, ${ }^{2}$ K. Banan Ali-Abasi, \\ ${ }^{2}$ A. Ghahramani and ${ }^{3}$ E. Amini \\ ${ }^{1}$ Power Engineering Department, Faculty of Electrical and Computer Engineering, \\ University of Tabriz, Tabriz, Iran \\ ${ }^{2}$ Azarbaijan Higher Education and Research Center Power Ministry, Tabriz, Iran \\ ${ }^{3}$ Azarbaijan Regional Electric Company, Tabriz, Iran
}

\begin{abstract}
Calculation of Current Transformers (CTs) magnetic and thermal properties are very complex due to the complexity of their construction, different properties of their materials and nonlinearity of core B-H curve. Finite Element Methods (FEMs) are very capable and reliable methods for these problems solution, such as Ansys software. In this study Ansys software is applied in analysis of an 800-400/5-5 CT. These analyses consist of 2D static normal, open circuit and short circuit condition of CT. Magnetic and thermal analysis are made and the results will be discussed.
\end{abstract}

Key words: Current transformer, magnetic analysis, thermal analysis, ansys software

\section{INTRODUCTION}

Modern protective systems require a faithful reproduction of primary short circuit current. Often, specially, in high power installations an important part of the current during a few cycles at least is the DC component, which causes severe saturation conditions, if the current transformer is not correctly selected and employed. Due to the importance of a good response of the measurement and protection systems in the reliability of the power systems, it is necessary to study of the CTs in any conditions as good as possible.

It is important to be able to determine the behavior of a CT within a certain range of accuracy when it is applied a primary current, since this will allow predicting the behavior of related equipment, such as that aimed at protecting power electric systems, which due to this situation might make an incorrect operation within the period involved.

Many analyzes have been presented to shows the CT behavior, but only some of them are well suited for transient conditions. FEMs are one of the best and suitable tools for this purpose $\mathrm{e}^{[2,3]}$. One-stage ferromagnetic current transformer is a kind of device which keeps a steady-state measurement error ${ }^{[4]}$. In this study for transient conditions and low error calculations, Ansys software is used for analysis of a medium voltage CT $800-400 / 5-5$.
Ct nominal values and physical dimensions: Using the Ansys Parametric Design Language (APDL), the physical dimensions and any necessary nominal and condition values of the used model are defined in the software environment. Table 1 shows the nominal ratings of this model ${ }^{[5,6]}$. In Fig. 1 geometrical model and physical dimensions of the CT are shown. Fig. 2 shows the magnetizing curve of the CT core.

Table 1: Nominal Ratings Of The Ct

\begin{tabular}{ll}
\hline Voltage & $72.5 \mathrm{kV}$ \\
Power & $75 \mathrm{VA}$ \\
Frequency & $50 \mathrm{~Hz}$ \\
Current ratio & $800-400 / 5-5$ \\
Maximum temperature & 80 times the nominal current \\
Support & in $1 \mathrm{sec}$ \\
\hline
\end{tabular}

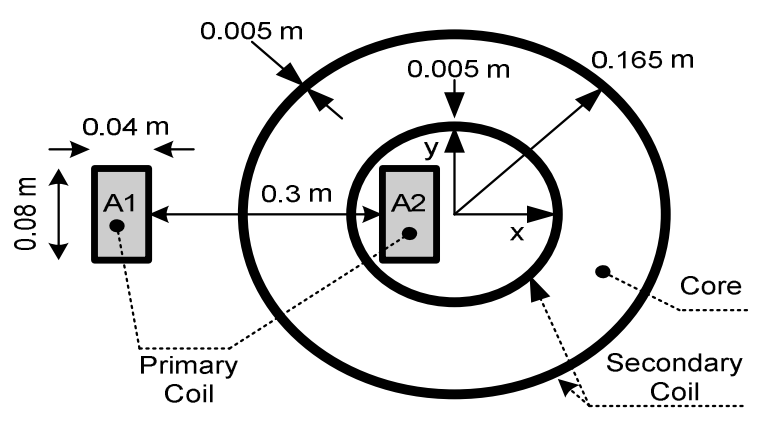

Fig 1: Geometrical model of the CT

Corresponding Author: M.B.B. Sharifian, Power Engineering Department, Faculty of Electrical and Computer Engineering, University of Tabriz, Tabriz, Iran 


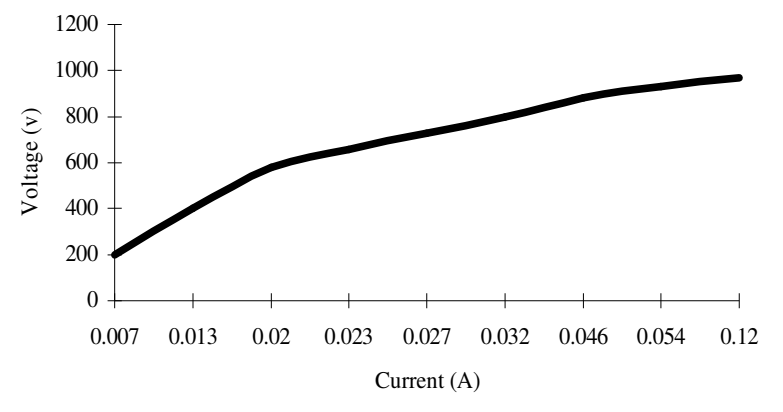

Fig. 2: The saturation curve of used CT

Magnetic analysis: Necessary parameters in the magnetic analysis are as follows:

- Geometrical view of the model

- Core B-H curve

- Primary and secondary currents densities

- Air, oil and windings magnetic properties

After the model geometrical view definition, first of all types of elements must be define. In the second step material properties of core, windings and other parts of the CT must be added to the model. In the third step meshing of the model can be made.

Thermal analysis: Coupling of different fields is one of the abilities of the Ansys software. This can be exerting in two cases:

- Direct coupling

- Indirect coupling

In the first case, two fields are coupled together directly and the results of the first field are applied as the load or input of the second field. In the second case both of the fields are analyzed separately. Results of the first field are stored in a file and recalling at the required time during the second field analysis as a load or input. Direct coupling are made for simple analysis, but for simplex analysis it take a long time for convergence, while it has error. In these conditions, using the indirect coupling can be very comfortable ${ }^{[1]}$.

In this study for simplicity direct coupling of electrical and thermal fields are used. Output of the electrical analysis is directly inputted to the thermal analysis as the load. Necessary parameters in the thermal analysis are as follows:

- Thermal conductance coefficient of any parts of the model
- Thermal transmission coefficient of any parts of the model, $\mathrm{h}$ can be calculated analytically in some apparatuses

- Primary windings resistance

- Oil temperature in the surface, or ambient temperature

\section{RESULTS AND DISCUSSION}

Magnetic analysis in normal condition: Figure 3 and 4 show the 2D flux distribution and flux density of the model in the normal condition analysis. Maximum value of the flux density is about $0.16198 \mathrm{~T}$, that is occur in the inner side of the coreandat the outer side of the core flux density distribution have the minimum value of the core. Figure 5 shows field intensity distribution in the model. Field intensity has its maximum about $8867 \mathrm{~A} / \mathrm{m}$ around the primary windings. In Fig. 6 2D distribution of electromagnetic forces of the model are shown in the normal condition analysis. Maximum of the forces, about $1.353 \mathrm{~N}$, are again around the primary windings.

Magnetic analysis in the open circuit condition: Figure 7 and 8 shows the 2D flux distribution and flux density of the model in the open circuit condition analysis. Due to the saturation of the core flux density in the core is very high. Maximum value of the flux density is about $1.631 \mathrm{~T}$ in the core. In Fig. 9 field intensity distribution of the model in the open circuit condition is shown. Field intensity around the primary winding has its maximum about $8719 \mathrm{~A} / \mathrm{m}$. In Fig. 10 2D distribution of electromagnetic forces of the model are shown in the open circuit condition analysis. Maximum of the forces, about $1.429 \mathrm{~N}$, are again around the primary windings.

Magnetic analysis in the short circuit condition: In this case, operation of the $\mathrm{CT}$ in a short circuited system is studied and analyzed. In these conditions CT is not in normal conditions and different tensions can be affects it, which must be analyzed accurately. Short circuit current is about 20 times the nominal vale, with respect to the short circuit level of the system.

Figure 11 and 12 show the flux distribution and flux density of the model in this case. Maximum flux density is about $1.544 \mathrm{~T}$.

In Fig. 13 distribution of electromagnetic forces of the model are shown, with a maximum value about $541.401 \mathrm{~N}$ around the primary winding. In these conditions these forces are very high and can be destroyed the CT due to the produced electrodynamics and mechanical tensions. 

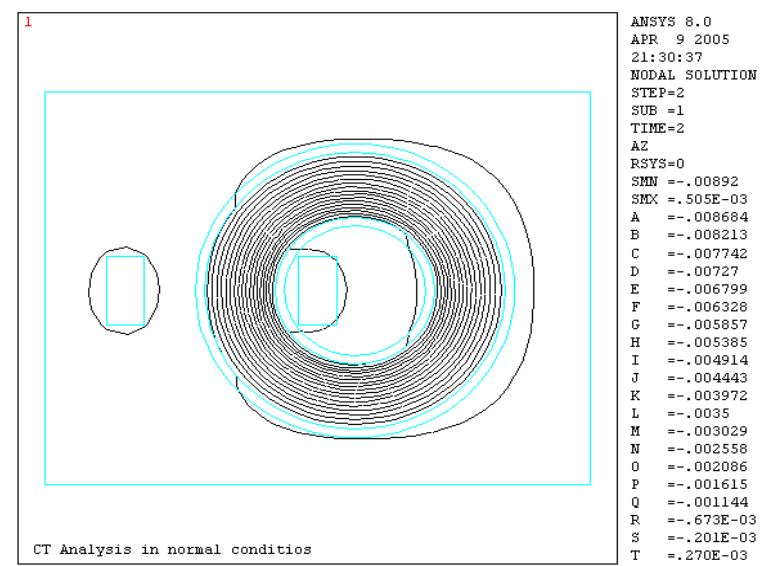

Fig. 3: 2D flux distribution in normal condition

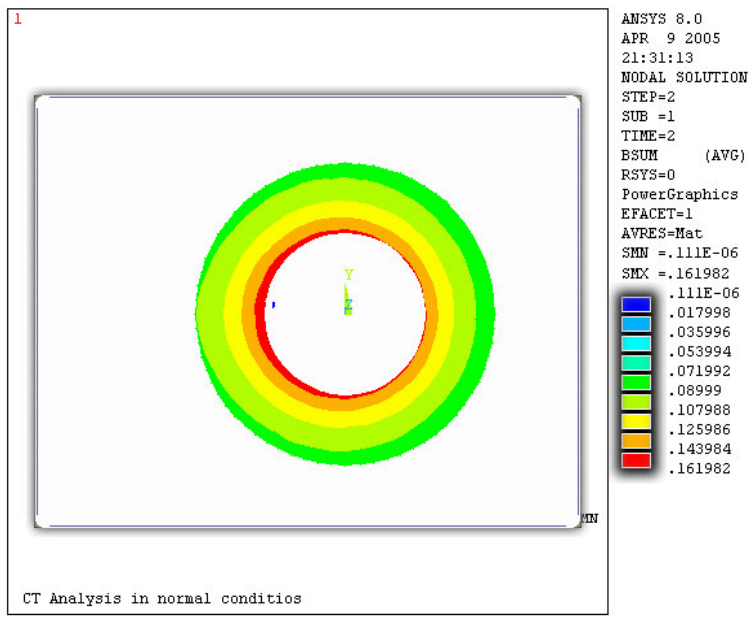

Fig. 4: 2D flux density distribution in normal condition

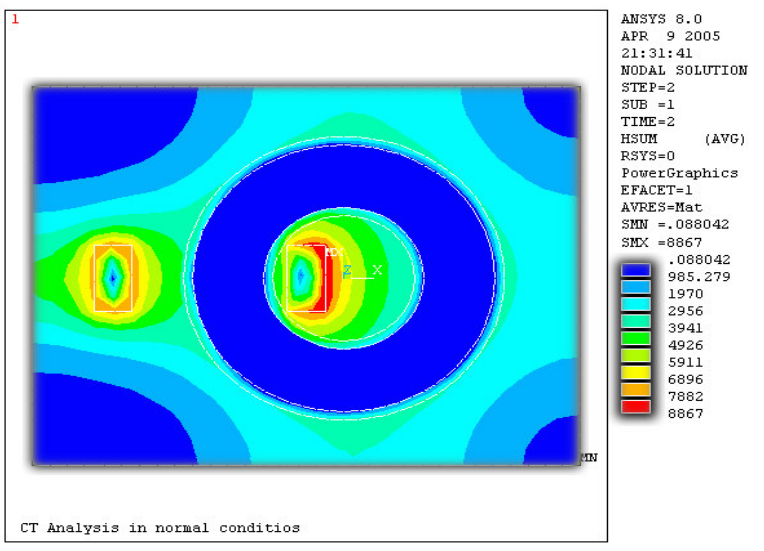

Fig. 5: 2D field intensity distribution in normal condition

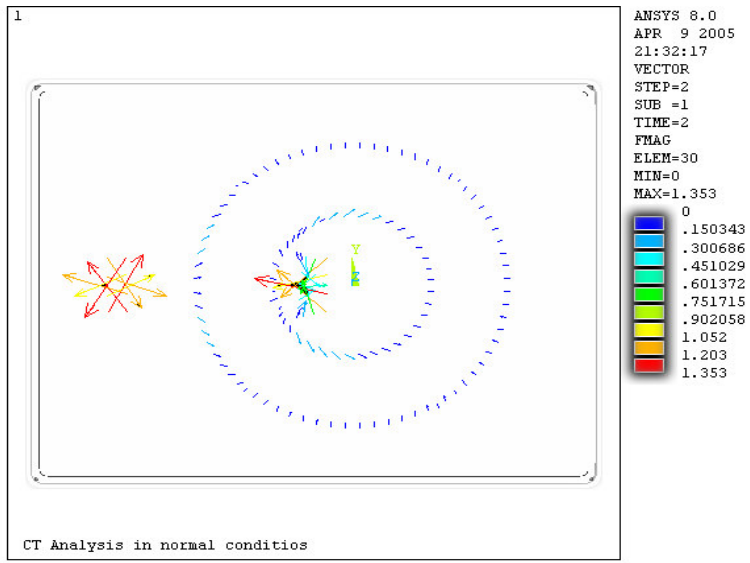

Fig. 6: Electromagnetic force distribution in normal condition
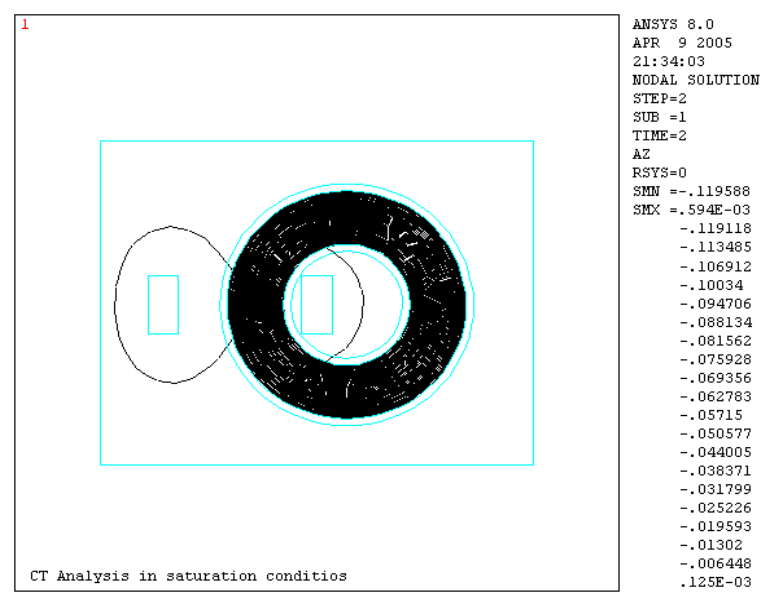

Fig. 7: 2D flux distribution in open circuit condition

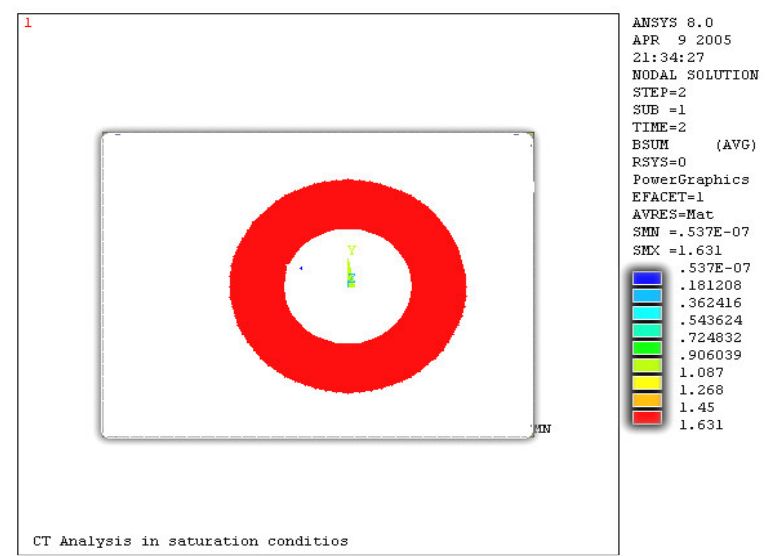

Fig. 8: 2D flux density distribution in open circuit condition 


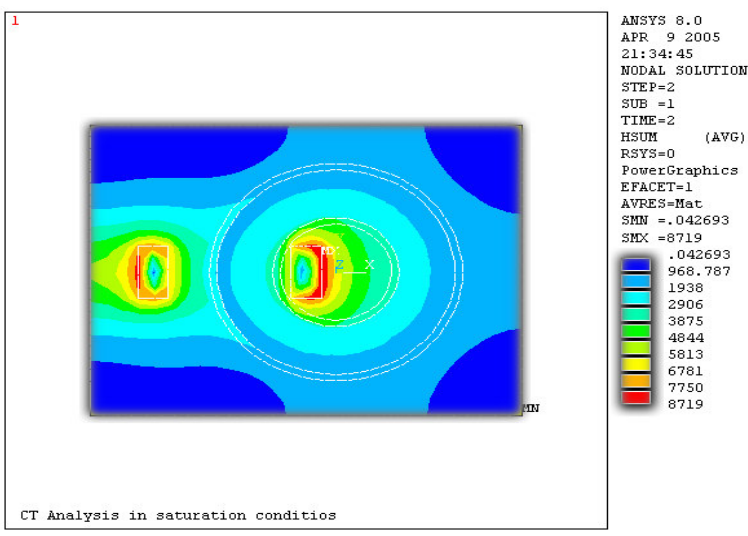

Fig. 9: 2D field intensity distribution in open circuit condition

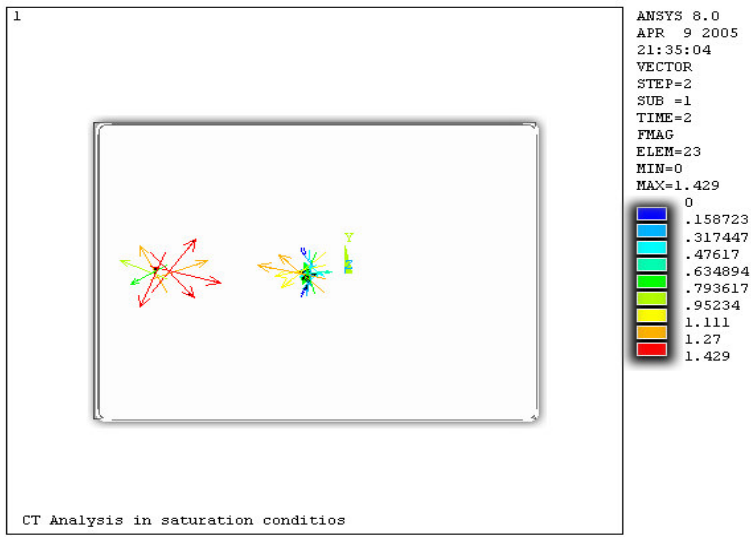

Fig. 10: Electromagnetic force distribution in open circuit condition

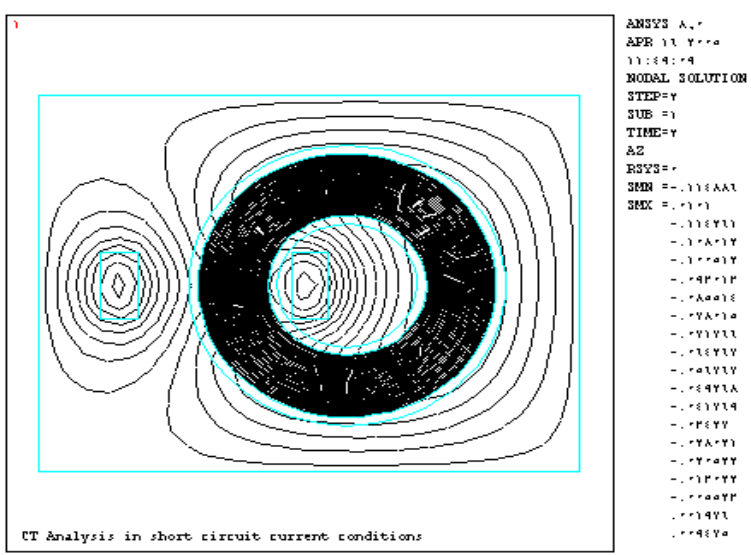

Fig. 11: 2D flux distribution in short circuit condition

Thermal analysis in the normal condition: Figure. 14 shows thermal distribution of the model in this case.

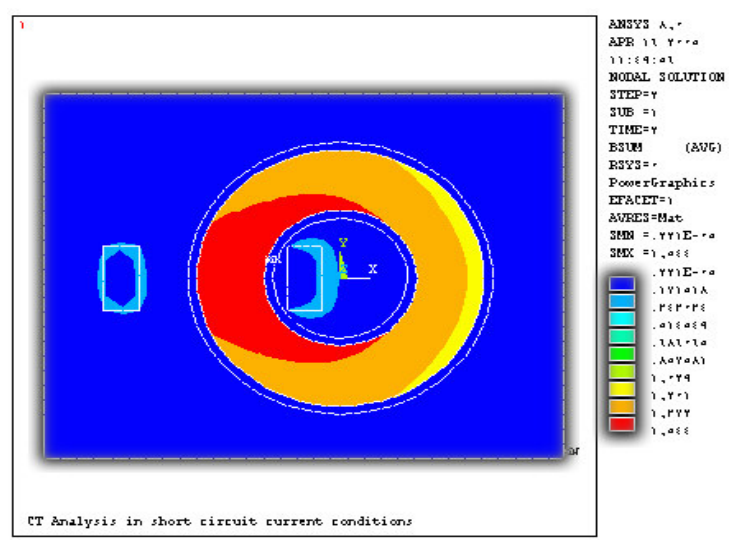

Fig. 12: D flux density distribution in short circuit condition

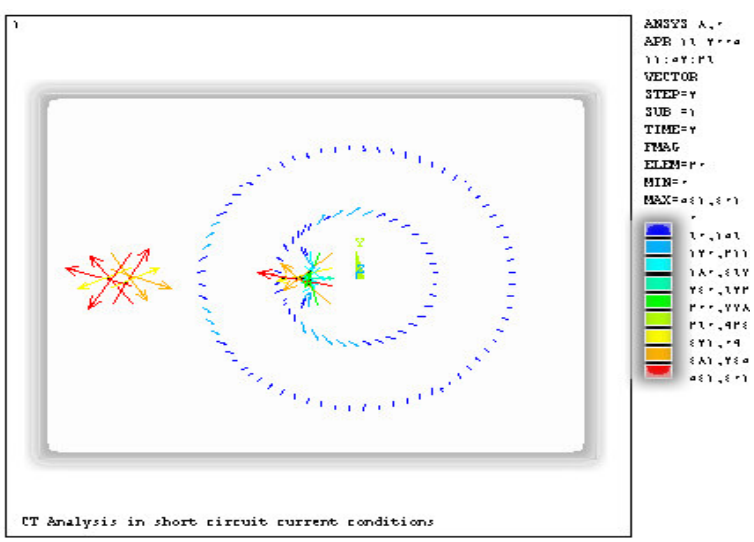

Fig. 13: Electromagnetic force distribution in short circuit condition

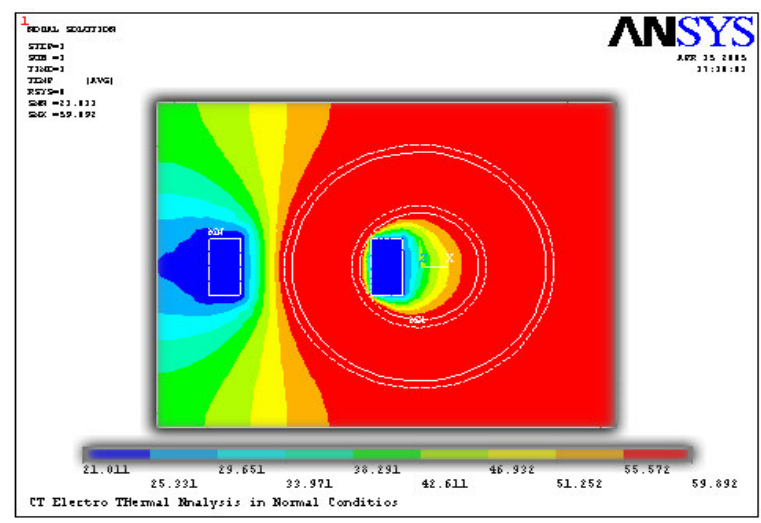

Fig. 14: Thermal distribution in normal condition

Around the secondary winding temperature rise is high, about $59.894^{\circ} \mathrm{C}$. Around the primary winding temperature rise is about $21.011^{\circ} \mathrm{C}$. 


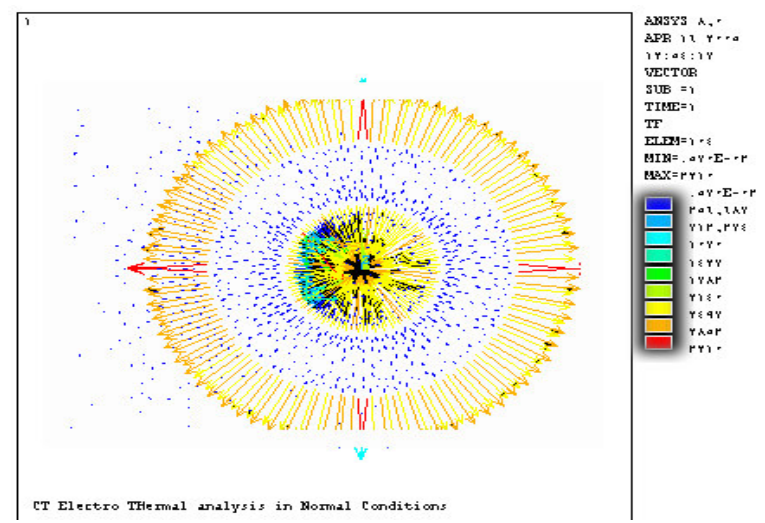

Fig. 15: Thermal flux distribution in normal condition

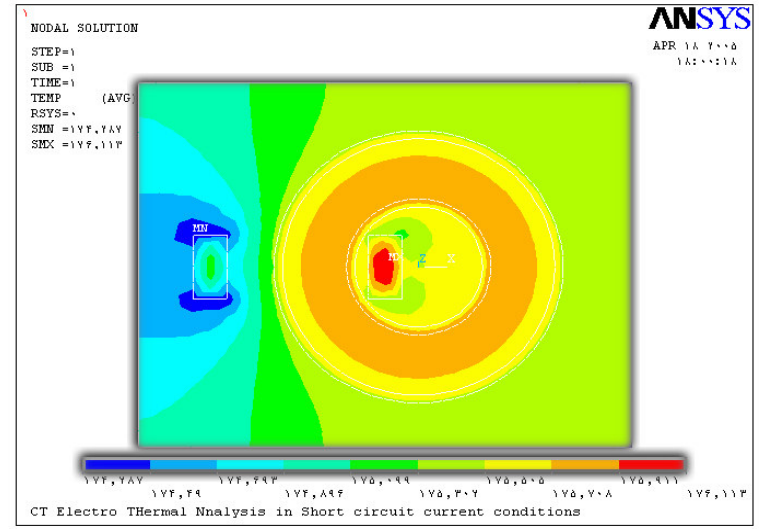

Fig. 16: Thermal distribution in short circuit condition

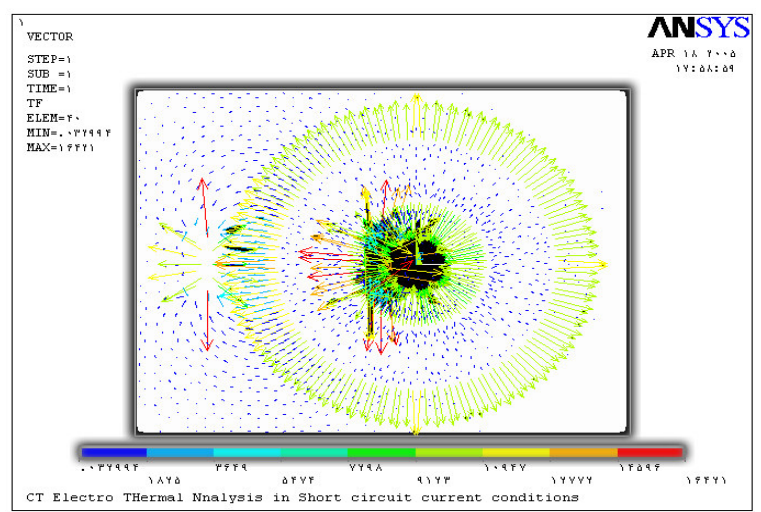

Fig. 17: Thermal flux distribution in short circuit condition

Figure. 15 show the thermal flux distribution of the model during this condition. In fact this figure shows the heat production sources and thermal distribution directions, from core to the oil. Maximum production of heat is in due to the secondary winding.
Thermal analysis in the short circuit condition: In Fig. 16 thermal distribution of the model during a short circuit in the system is shown. It shows that temperature rise around the primary winding is high, about 159.629 $\mathrm{C}$ and around the secondary winding is low, about 61.727 C. In fact heat production of the secondary winding is lower than that of primary winding.

Figure. 17 show thermal flux production and distribution in the model during this condition. Maximum heat production is due to the primary winding of the CT.

\section{CONCLUSIONS}

In this research magnetic and thermal analysis of a $\mathrm{CT}$ is down using 2D FEM. Ansys software is chosen for analysis. Different operating conditions of the system are studied.

In the normal condition that the primary and secondary windings of the CT carries the nominal currents, maximum flux density, maximum field intensity and maximum electromagnetic force are $0.16198 \mathrm{~T}, 8867 \mathrm{~A} / \mathrm{m}$ and $1.353 \mathrm{~N}$ respectively. In the case that the secondary of the CT is open circuited, maximum flux density, maximum field intensity and maximum electromagnetic force are $1.631 \mathrm{~T}, 8719 \mathrm{~A} / \mathrm{m}$ and $1.429 \mathrm{~N}$ respectively. So flux density is very larger than that of the normal condition and core will be saturated.

In the case that a short circuit is occurred in the system, as an example with amplitude of 20 times the normal current, electromagnetic forces can be increased to about $541.401 \mathrm{~N}$. These large electromagnetic forces can produced larger electrodynamics tensions and destroy the CT. Using a coupled magnetic and mechanical field analysis we can specify the weak and strong points of the CT from mechanical point of view.

In the normal operating condition, the maximum temperature rise is about $59.894 \square \mathrm{C}$ and in the short circuit case, this value increases to the $173.113 \square$ C, which is a high temperature for the oil and can be change the dielectric and cooling properties of the CT oil.

\section{REFERENCES}

1. Ansys Software Documents, Version 9.0, 2004.

2. Shackelford, J.F., Introduction to Material Science for Engineers, Maxwell Macmillan Pub. Co., New York, 1992.

3. Vanvlack, L H., 1982. Materials for Engineering: Concepts and Applications, Addison Wesley. 
4. Hai, B., A. Xin and L. Huifang, 2002. A New Active Compensation for Phase Error of Current Transformer Basing on Self-Adaptive Control Method, Power Systems and Communications Infrastructures for the future, Beijing, September.

5. Fakhri A., 2004. Thermal Modeling of Transformer Windings using Magnetic and Thermal Fields Coupling in FEM Environment, MSc Thesis, University of Tabriz, Iran.
6. Ghahramani, A., 2000. Study and Modeling of Current Transformers Saturation using EMTPandProduction of a Suitable Method for Secondary Current Comparison in this Condition, MSc. Thesis, University of Tabriz, Iran. 